\title{
Tuberculosis diagnosis in resource-limited settings: Clinical use of GeneXpert in the diagnosis of smear-negative PTB: a case report
}

\author{
*Nakiyingi $\mathrm{L}^{1,2}$, Nankabirwa $\mathrm{H}^{1}$, Lamorde $\mathrm{M}^{2}$
}

1. Department of Medicine, College of Health Sciences, Makerere University, Kampala, Uganda.

2. Research Department, Infectious Diseases Institute, College of Health Sciences, Makerere University, Kampala Uganda

\begin{abstract}
Background: The Xpert MTB/RIF test (GeneXpert) has recently been endorsed for use in resource-limited settings for the diagnosis of tuberculosis and drug resistant-tuberculosis. In resource-limited settings, GeneXpert has been used predominantly for research and there is little experience with its use in day-to-day management of patients.

Case summary: We describe a case of a 46 year old HIV-infected male with smear-negative pulmonary tuberculosis, who had several visits to various lower level health centres and two admissions in a tertiary care hospital; however, the diagnosis of tuberculosis was only made several months later on GeneXpert testing that was performed under a research project.

Conclusion: GeneXpert facilitated identification of tuberculosis in an HIV positive patient in whom the diagnosis had been delayed when more widely available tests were used. Operational and cost-effectiveness studies are needed to provide evidence to policy makers in order to improve access to GeneXpert.
\end{abstract}

Key words: Tuberculosis; developing countries; molecular diagnostic techniques.

African Health Sciences 2013; 13(2): 522 - 524 http://dx.doi.org/10.4314/ahs.v13i2.46

\section{Introduction}

Early treatment of tuberculosis (TB) is hindered by the lack of rapid, accurate diagnostic modalities that can be applied in resource-limited settings (RLS ${ }^{1-2}$. Sputum smear microscopy ${ }^{1}$ which is the cheapest and the most available method of TB diagnosis identifies TB in less than half of patients with HIV/ TB co-infection. Access to mycobacterial culture is limited and where available results are often delayed by several weeks. Xpert MTB/RIF test (GeneXpert) is a promising innovation in routine TB diagnosis in developing countries owing to the its high sensitivity, specificity and rapid turnaround time of only two hours $^{3-6}$. GeneXpert is an integrated fully automated specimen processing and nucleic-acid-amplification test $^{3-4}$ that was recently endorsed for use in RLS by the World Health Organization for the diagnosis of MTB and drug resistant-TB. However, its roll-out is hampered by the relatively high cost of the machine, need to import diagnostic cartridges and need for constant and stable electricity supply ${ }^{5}$. We present a

\section{*Corresponding author:}

Dr. Lydia Nakiyingi

Department of Medicine

Makerere University, College of Health Sciences

P. O. Box 7072, Kampala, Uganda

Tel: +256 772468045

E-mail: lydikiyingi@yahoo.com case highlighting the clinical use of this test in a high burden TB setting.

\section{Case summary}

A 46 year old Ugandan male truck driver presented to the medical wards of Mulago National Referral Hospital in January 2012 with productive cough, low grade fevers and left-sided chest pain of six weeks' duration. Prior to this visit, he had made several visits to health providers in lower level health centres for the same illness and received unknown oral antibiotics with no improvement. He had no previous history of TB and was a non-smoker. His HIV serology test was positive. Although smear microscopy examinations performed on two sputum samples were negative for acid fast bacilli (AFB), his chest radiograph revealed consolidation in the left mid and lower lung zones (figure 1a). He was treated for bacterial pneumonia with cephalosporins (ceftriaxone 1gram given intravenously at 12 hourly for 5 days), followed by a macrolide (azithromycin tablets $500 \mathrm{mg}$ every 24 hours for 3 days) and discharged a week later on cotrimoxazole prophylaxis, referred for HIV care and scheduled for follow-up one month later. However, three weeks after discharge, he returned to hospital with worsening cough, fever, weight loss and anorexia. On examination, he was febrile (temperature $37.9 \mathfrak{x} \% \mathrm{C}$ ), wasted (body mass index 
$=16.2 \mathrm{~kg} / \mathrm{m} 2)$, dehydrated and pale. He was tachypneic (respiratory rate 30 breathes/min) and still had features of left consolidation.

Laboratory investigations findings included significantly low CD4 count (21cells/ $\mu \mathrm{l})$, raised ESR $(80 \mathrm{~mm} / \mathrm{hr}$ ), mild leucopenia (WBC count $3.42 \mathrm{X} 103 \mathrm{cells} / \mu \mathrm{l})$ with normal differential counts, normochromic anemia $(9.2 \mathrm{~g} / \mathrm{dl})$, hypoalbuminemia $(18.2 \mathrm{~g} / \mathrm{l})$ and normal serum chemistries. His chest radiograph (figure $1 \mathrm{~b}$ ) revealed signs of persisting consolidation but repeat smear microscopy exams were negative for AFB. Bacterial blood cultures were negative, mycobacterial cultures were not performed as they are not part of the routine TB diagnostic work up for this hospital. Abdominal ultrasound scan showed micro liver abscesses.

The patient showed no changes in signs and symptoms after 2 weeks on admission and treatment with intravenous fluids and antibiotics which included ceftriaxone 1gram given intravenously every twelve hours daily for one week. GeneXpert testing was performed under a research project (since the test was not readily available in this hospital) and this detected mycobacterium tuberculosis that was sensitive to Rifampicin. The patient was commenced on antiTB therapy in accordance with Uganda guidelines and he showed significant improvement. He was discharged a week later and subsequently commenced antiretroviral therapy (tenofovir disoproxil fumarate, emtricitabine plus efavirenz). The patient continues to be well.

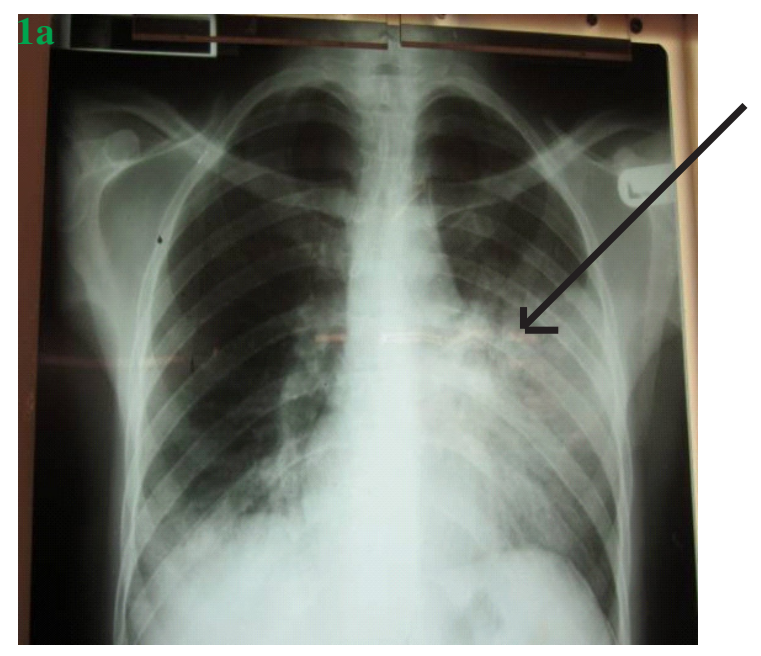

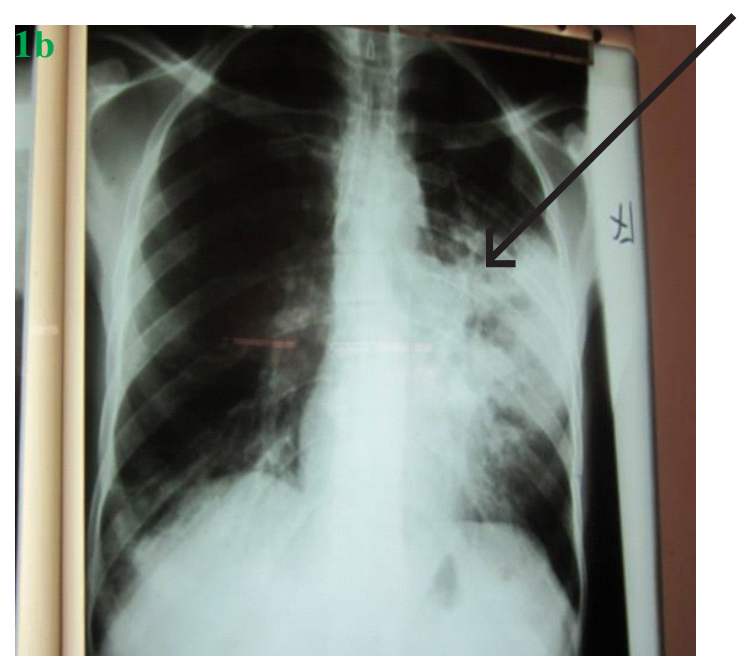

Figure 1: Chest radiographs taken one month apart showing consolidation (arrow) involving the left lower and mid lung zones

\section{Discussion}

This case illustrates the diagnostic difficulties encountered in the management of sputum smearnegative TB in a setting of high HIV/TB prevalence and the clinical benefit of GeneXpert. The HIV epidemic has led to an increase in the incidence of smear-negative pulmonary TB and such cases are increasingly common ${ }^{1}$ in RLS. Smear-negative pulmonary TB is associated with poor treatment outcomes, including death due to delayed diagnosis or non-diagnosis ${ }^{1-2}$. New technologies like GeneXpert provide an opportunity for early identification of $\mathrm{TB}^{5,7,8,9}$ which could reduce morbidity and mortality in smear- negative TB patients. GeneXpert is a promising new test in routine TB diagnosis in developing countries owing to its high sensitivity, specificity and rapid turnaround time ${ }^{3-}$ ${ }^{6}$. It is an integrated specimen processing and nucleicacid-amplification test which is fully automated and offers results in only two hours ${ }^{3-4}$. In all settings, GeneXpert test has consistently demonstrated better performance $^{5-8}$ when compared to smear microscopy and its performance compares well with liquid culture. The assay is also highly specific since there is no cross-reaction with non-tuberculous mycobacteria ${ }^{3-4}$. Furthermore, the performance of GeneXpert is not affected by HIV co infection ${ }^{6}$. In a multi-country study ${ }^{6}$ (including Uganda), the sensitivity of GeneXpert testing was $90 \cdot 3 \%$ among culture-confirmed TB cases, $76 \cdot 9 \%$ among smearnegative, culture-positive TB patients and the specificity was $99 \cdot 0 \%$. 
The cost of a GeneXpert test compares favourably to mycobacterial culture but it has the distinct advantage of a faster turn-around time, providing same-day diagnosis which could potentially limit loss to follow up during diagnostic evaluation of smear- negative TB patients ${ }^{2,6,9}$. Furthermore, GeneXpert testing implementation may be cost saving both to the patient and the health system ${ }^{5,6,9}$. GeneXpert testing could potentially reduce the costs associated with clinic visits, drugs, hospital admissions and chest $\mathrm{x}$-rays as well as TB associated morbidity and mortality. From the health system point of view, implementation of GeneXpert testing could potentially reduce physician consultations, hospital bed occupancy, transmission and antibiotic use. However, formal cost-effectiveness studies on GeneXpert have not been conducted in Uganda.

Mycobacterial cultures are the gold standard for TB diagnosis but were not performed for the patient in this report because this is not standard of care for new TB suspects at our centre. Culture results are often obtained several weeks after samples are collected and therefore, a diagnostic delay would also be expected with this method.

\section{Conclusion}

GeneXpert facilitated identification of TB in this HIV positive patient in whom the diagnosis had been delayed when more widely available tests were used. However, operational and cost-effectiveness studies are needed within the local context to support allocation of resources to expand access to innovative technologies like GeneXpert.

\section{Consent}

Informed consent was obtained from the patient for publication of this case report and any accompanying images.

\section{Acknowledgments}

Makerere University Tuberculosis laboratory for performing the GeneXpert testing, the patient who permitted the write up, the clinical team of the pulmonology unit of Mulago medical ward and the Infectious Diseases Institute, Makerere University for the scientific support.

\section{References}

1. Getahun H, Harrington M, O'Brien R, Nunn P. Diagnosis of smear-negative pulmonary tuberculosis in people with HIV infection or AIDS in resource-constrained settings: informing urgent policy changes. Lancet. 2007;369(9578):2042-9.

2. Lawn SD, Wood R. Tuberculosis in antiretroviral treatment services in resource-limited settings: addressing the challenges of screening and diagnosis. J Infect Dis. 2011;204 Suppl 4:S115967.

3. Helb D, Jones M, Story E, Boehme C, Wallace E, Ho K, et al. Rapid detection of Mycobacterium tuberculosis and rifampin resistance by use of on-demand, near-patient technology. J Clin Microbiol. 2010;48(1):229-37.

4. Blakemore R, Story E, Helb D, Kop J, Banada P, Owens MR, et al. Evaluation of the analytical performance of the Xpert MTB/RIF assay. $J$ Clin Microbiol. 2010;48(7):2495-501.

5. Van Rie A, Page-Shipp L, Scott L, Sanne I, Stevens W. Xpert((R)) MTB/RIF for point-ofcare diagnosis of TB in high-HIV burden, resource-limited countries: hype or hope? Expert Rev Mol Diagn. 2010;10(7):937-46.

6. Boehme CC, Nicol MP, Nabeta P, Michael JS, Gotuzzo E, Tahirli R, et al. Feasibility, diagnostic accuracy, and effectiveness of decentralised use of the Xpert MTB/RIF test for diagnosis of tuberculosis and multidrug resistance: a multicentre implementation study. Lancet. 2011 Apr 30;377(9776):1495-505.

7. Lawn SD, Brooks SV, Kranzer K, Nicol MP, Whitelaw A, Vogt M, et al. Screening for HIVassociated tuberculosis and rifampicin resistance before antiretroviral therapy using the Xpert MTB/RIF assay: a prospective study. PLoS Med. 2011 Jul;8(7):e1001067.

8. Rachow A, Zumla A, Heinrich N, Rojas-Ponce G, Mtafya B, Reither K, et al. Rapid and accurate detection of Mycobacterium tuberculosis in sputum samples by Cepheid Xpert MTB/RIF assay-a clinical validation study. PLoS One. 2011;6(6):e20458.

9. Lawn SD, Nicol MP. Xpert(R) MTB/RIF assay: development, evaluation and implementation of a new rapid molecular diagnostic for tuberculosis and rifampicin resistance. Future Microbiol. 2011 Sep;6(9):1067-82. 